\title{
IMWG Sequencing MRD-negative
}

National Cancer Institute

\section{Source}

National Cancer Institute. IMWG Sequencing MRD-negative. NCI Thesaurus. Code C159802.

Absence of clonal plasma cells by next-generation sequencing on bone marrow aspirate in which presence of a clone is defined as less than two identical sequencing reads obtained after DNA sequencing of bone marrow aspirates using the LymphoSIGHT platform (or validated equivalent method) with a minimum sensitivity of 1 in $10^{\wedge} 5$ nucleated cells or higher. 